\title{
Comparison effectiveness between cetylpyridinium chloride and triclosan mouthwash on plaque
}

\author{
Gurtej Singh Chinger*, Dede Hadidjah*, Nunung Rusminah* \\ *Department of Periodontics Faculty of Dentistry Universitas Padjadjaran
}

\begin{abstract}
Introduction: Dental plaque is a soft deposit that accumulates on the teeth. It is a complex microbial community with as many as 400 distinct bacterial species. One of the most prevalent diseases of the oral cavity is periodontal disease which begins with gingivitis. The main cause of gingivitis is plaque accumulation. Aim of this research is to comparison effectiveness of mouthwash containing cetylpyridinium chloride and mouthwash containing triclosan on plaque. Methods: The research was experimental study, with the double blinded parallel design. A total of 30 students of SMA Pasundan 8 were chosen. Clinical examination was conducted to obtain the plaque score using the Quigley Hein Index (modified) 1970. Subjects were divided into two groups. One group was given the cetylpyridinium chloride mouthwash and the other group was given triclosan mouthwash. Data were then analysed using the ANOVA test. Results: there is no significant difference between the effectiveness of mouthwash containing cetylpyridinium chloride and mouthwash containing triclosan on plaque $(P>0.05)$. Conclusion: the mouthwash containing cetylpyridinium chloride and mouthwash containing triclosan is effective in reducing plaque.
\end{abstract}

Keywords: Cetylpyridinium chloride, triclosan, Quigley Hein index.

\section{INTRODUCTION}

Basically, health is very essential for human being and oral health is a part of general health. Oral hygiene is associated with the occurrence of gum and periodontal disease, the lower the level of cleanliness of one's mouth, it will be more easy for periodontal and gum disease to occur. Periodontal disease is a term which includes all pathological conditions of the gingiva, cementum, periodontal membrane and alveolar bone.

It is however used in reference to those lesions which have an inflammatory basis and are described as gingivitis or periodontitis ${ }^{1,2,3}$ Maintaining oral hygiene is to control plaque which is needed for prevention and treatment of gingivitis and periodontitis. Plaque can be defined as biofilm, usually pale yellow, that develops naturally on the teeth. There are 2 methods of controlling plaque which can be done by chemical and mechanical.

Mechanical plaque control is the removal of microbial and the prevention of accumulation on the teeth and adjacent gingival surface by the use of tooth brush and other mechanical hygiene aids. Plaque control using chemical way is one of the most common way used by the community. Chemical agents acts on the plaque ultrastructurally and prevent the formation of plaque. Plaque removing efforts can be done 
through chemical using mouthwash. Mouthwash is a kind of solution which is used to clean the oral cavity. Some mouthwash comes with anti-plaque properties which can kill the bacterial plaque.

Cetylpyridinium Chloride (CPC) is a cationic quaternary ammonium compound in some types of mouthwashes. It is an antiseptic that kills bacteria and other microorganisms. Alcohol has an extensive history of safety when used in products as recommended, for the vast majority of the population. However, certain individuals may not be able to tolerate alcohol-containing mouthrinses for a variety of reasons, so there are choices available to be recommended to our patients. CPC has shown to besafe. In a mouthwash, it binds to the surface of the germs and causes them to burst, which helps reduce their build up on the teeth ${ }^{4}$.

Triclosan is an antibacterial agent used in mouthwash. It is a polychloro phenoxy phenol and an agent that is effective in reducing and controlling bacterial growth. Triclosan (2,4,4'-trichloro2 'hydroxydiphenyl ether) is used to increase the ability of mouthwash to bind to the oral mucosa and thus be available for longer periods of time. This organic compound is a white powdered solid with a slight aromatic and phenolic odor. It is a chlorinated aromatic compound that has functional groups representative of both ethers and phenols. Phenols often show antibacterial properties 5 .

Based on the above information, therefore the writer was interested to do this research on comparison between Cetylpyridinium Chloride and Triclosan Mouthwash on Plaque on students of SMA Pasundan 8, Bandung. We encouraged all SMA Pasundan 8 school student who was undergoing treatment in RSGM. Since there was limited dental services around the school area, we encouraged to boost the level of awareness of oral hygiene to the students of SMA Pasundan 8. Aim of this research is to comparison effectiveness of mouthwash containing cetylpyridinium chloride and mouthwash containing triclosan on plaque.

\section{METHODS}

The type of research was experimental, with the double blinded parallel design. A total of 30 students of SMA Pasundan 8 were chosen. The criteria of the volunteers are as follows: male or female, in good general health, free from any medication, does not wear any orthodontic or prosthodontic appliance, willing to be a subject of research (informed consent).

Clinical examination was conducted to obtain the plaque score using the Quigley Hein Index (modified) 1970. Subjects were divided into two groups. One group was given the cetylpyridinium chloride mouthwash and the other group was given triclosan mouthwash. Data were then analysed using the ANOVA test. For this study the plaque score is taken on the Ramfjord teeth.

\section{RESULTS}

From the table 2 , it shows that all the results are significant $(\mathrm{Sig}=0.000)$ means there is difference in reducing plaque after using mouth-

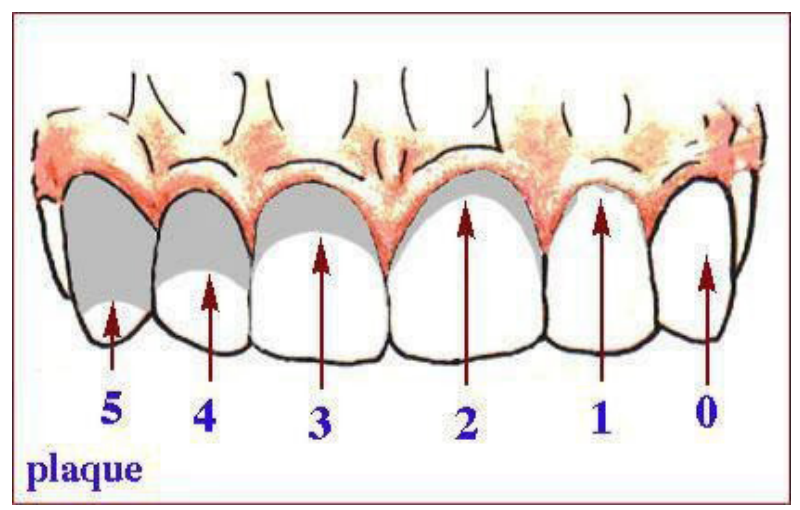

Figure 1. Quigley-hein plaque index

Table 1. Scoring criteria for plaque index quigely hein index (modified) 1970

0 No plaque

1 Separate fleck of plaqie (up to one $\mathrm{mm}$ ) at the cervical margin of the tooth

2 A thin continuous band of plaque wider than $1 \mathrm{~mm}$ but covering less than one0third of the crown of the tooth

3 A band of plaque wider than one mm but covering less than one-third of the crown of the tooth

4 Plaque covering at least one-third but less than two-thirds of the crown of the tooth

5 Plaque covering two-thirds or more of the crown of the tooth 


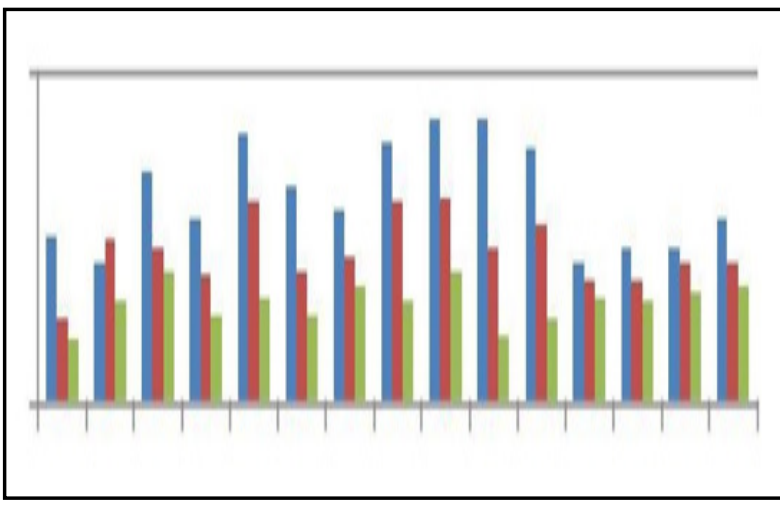

Figures 2 graph shows the distribution of plaque accumulation obtained for group cetylpyridinium chloride

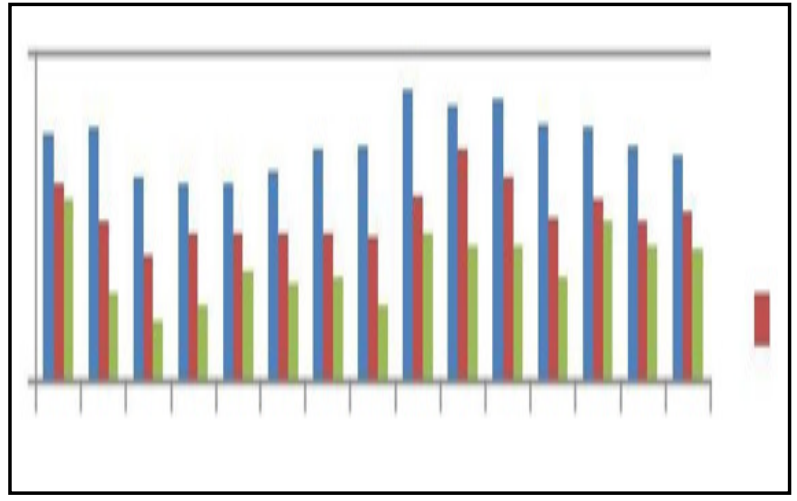

Figures 3 graph shows the distribution plaque accumulation obtained for triclosan

Table 2. Comparison test for the amount of plaque according to time for cetylpyridinium chloride and triclosan

\begin{tabular}{lrrrrrr}
\hline \multirow{2}{*}{ Mouthwash } & \multicolumn{2}{c}{ Initial Check up - 3rd } & \multicolumn{2}{c}{ Initial Chek up- 7th } & \multicolumn{2}{c}{ 3rd Day-7th day } \\
\cline { 2 - 8 } & Mean diff & Sig & Mean diff & Sign & Mean & Sig \\
\hline Cetylpyridinium Chloride & 0,059722 & 0.000 & 0,084028 & 0.000 & 0,052778 & 0.001 \\
Triclosan & 01.09 & 0.000 & 0,101389 & 0.000 & 0,053472 & 0.000 \\
\hline
\end{tabular}

${ }^{*}$ The mean difference is significant at the 0.05 Level

Table 3. Comparison between cetylpyridinium chloride and triclosan mouthwash according to plaque scoring at initial check up

\begin{tabular}{cccccc}
\hline Mouthwash & $\begin{array}{c}\text { Mean plaque } \\
\text { score }\end{array}$ & $\begin{array}{c}\text { Std } \\
\text { deviation }\end{array}$ & t-test & Mean differences & Significant \\
\hline Cetylpyridinium Chloride & 3.12 & 0.79 & -2.16 & -0.51 & 0.005 \\
Triclosan & 3.63 & 0.45 & & & \\
\hline & ${ }^{*}$ The mean difference is significant at the 0.05 Level
\end{tabular}

Table 4 comparison between cetylpyridinium chloride and triclosan mouthwash according to plaque scoring at 3rd day

\begin{tabular}{rccccc}
\hline Mouthwash & $\begin{array}{c}\text { Mean plaque } \\
\text { score }\end{array}$ & $\begin{array}{c}\text { Std } \\
\text { deviation }\end{array}$ & t-test & Mean differences & Significant \\
\hline Cetylpyridinium Chloride & 2.27 & 0.52 & -1.16 & -0.27 & -0.27 \\
Triclosan & 2.53 & 0.41 & & & \\
\hline
\end{tabular}

Table 5. comparison between cetylpyridinium chloride and triclosan mouthwash according to plaque scoring at 7th day

\begin{tabular}{rccccc}
\hline Mouthwash & $\begin{array}{c}\text { Mean plaque } \\
\text { score }\end{array}$ & $\begin{array}{c}\text { Std } \\
\text { deviation }\end{array}$ & t-test & Mean differences & Significant \\
\hline Cetylpyridinium Chloride & 1.51 & 0.31 & -1.67 & -0.26 & 0.105 \\
Triclosan & 1.77 & 0.52 & & & \\
\hline
\end{tabular}

washcontaining

Cetylpyridinium Chloride and mouthwash containing Triclosan. There is a significant difference between the initial check up with the 3rd day control and the 7th day controland also between the 3rd day control and the 7th day control while using the cetylpyridinium chloride mouth- wash and Triclosan mouthwash.

Results shows in table 5 that there is significant difference between mouthwash which contains Cetylpyridinium Chloride and Triclosan for Initial Check up, but there is no significant difference for 3rd day, and 7th day. (Table 3,4,5) 


\section{DISCUSSION}

The results of the research and statistical calculation show that mouthwash containing cetylpyridinium chloride and mouthwash containing triclosan have the same effectiveness against reducing plaque. During the 3rd day and the 7th day control for cetylpyridium chloride and triclosan, the result showed that the plaque has reduced. There was a previous research done by Karen BW to study the effects of a cetylpyridinium chloride mouthwash on gingivitis and plaque. The purpose of the following research was to evaluate the effects of mouth rinse containing $0.07 \%$ high bioavailable cetylpyridinium chloride on the development of gingivitis and plaque versus a placebo control ${ }^{6}$. The research's results showed that one 124 subjects were evaluable at Month 3 and 119 at Month 6 . After 6 months, subjects rinsing with the CPC rinse showed $15.4 \%$ less gingival inflammation, $33.3 \%$ less gingival bleeding, and $15.8 \%$ less plaque relative to the placebo group. All reductions were highly statistically significantly different $(P<0.01)$. Results were similar at 3 months.

Besides that, for triclosan, a randomized clinical evaluation of triclosan-containing dentifrice and mouthwash association in the control of plaque and gingivitis was carried out by Villalpando KT (2010). The aim of this doubleblind study was to evaluate the clinical efficacy of the association of triclosan-containing mouthwash and dentifrice on biofilm and gingivitis reduction. Intragroup evaluation revealed a statistically significant reduction in Plaque Index for groups that used triclosan). Intergroup analysis suggested that only the group that used the association of triclosan dentifrice and mouthwash (group TT) demonstrated statistically reduced plaque index at 30 days, when compared to the control group (CC) $(\mathrm{P}<.05)$. With regard to gingivitis reduction, no difference was observed between groups, although the association presented a faster reduction in bleeding levels. Only the association of triclosan dentifrice and triclosan mouthwash statistically reduced plaque index, when compared to the control group. However, regarding the additional benefits to gingivitis control, more studies should be done to confirm the results.

Another study was carried out by Wu $\mathrm{X}$
(2001) on effect of a new triclosan-containing mouthwash on oral infection. The objective was to study the effect of a new Triclosan-containing mouthwash--LIBO Anti-plaque mouthwash on oral infection?. As the results, Triclosan mouthwash was effective to decrease the plaque index (PLI) and supragingival bleeding index (SBI). Triclosan mouthwash has strongest anti-adhesion effect among the three tested agents and has continuous disinfecting effect and help control the formation and adhesion of dental plaque.

Based on the previous research above, it is proven that cetylpyridinium chloride and triclosan are indeed an effective antiseptic and antimicrobial. From the results of this research, initial check up to 3rd day control, both cetylpyridinium and triclosan mouthwash showed a reduction of plaque. Futhermore, from the 3rd day control to 7th day control, both cetylpyridinium chloride and triclosan showed reduction of plaque too. This research was only to see the effectiveness of mouthwash containing cetylpyridinium chloride and mouthwash containing triclosan towards reducing plaque.

\section{CONCLUSION}

Mouthwash containing cetylpyridinium chloride and mouthwash containing triclosan is effective in reducing plaque. Both of the mouthwash does not have clear difference in their effectiveness in reducing plaque.

\section{REFERENCES}

1. Moore LVH, Moore WEC, Cato EP, Smibert RM. Brumeiste JA. Bacteriology of human gingivitis. J Dent Res., 1987;66:989-995.

2. Wilkins $E$ M. Clinical Practice of dental Hygine. $8^{\text {th }}$ edition Philadelphia: Lippincott Williams \& Wilkins. 1999. p. 267-4.

3. Carranza, F.A.; Newman, M.G.; Takei, H.H.2002. Clinical Periodontology. 9th Edition. Philadelphia.: W.B Saunders Company. 96-101; 127.

4. Watanabe E. Dentistry, Dental Practice and the Community. $4^{\text {th }}$ ed. Philadelphia. W.B. Saunders Co. 2008.

5. Douglas JA. The Effective of Mouth Rinces. Available from:http://www. 
preventionmouthrinse.com. 2004.

6. Karen B 2005. Evaluation Cetylpyridinium chloride mouth rinse on gingivitis and plaque; Am J Dent 2005;18 (special issue):9A-14A
7. Wu X. Effect of a new triclosan-containing mouth rinse on oral infection; Department of Stomatology 2001;36(4):301-3. 